Delgado García, C.A. \& Méndez Romero, R.A. (2018). Políticas de integración en la educación universitaria. El caso de indígenas y afrocolombianos y el problema de la deserción. Revista Electrónica Interuniversitaria de Formación del Profesorado, 21(2), 109-123.

DOI: http://dx.doi.org/10.6018/reifop.21.2.323221

\title{
Políticas de integración en la educación universitaria. El caso de indígenas y afrocolombianos y el problema de la deserción
}

César Augusto Delgado García', Rafael Alberto Méndez Romero²

'Departamento de Matemáticas, Universidad del Valle

${ }^{2}$ Departamento de Matemáticas Aplicadas y Ciencias de la Computación, Universidad del Rosario

\section{Resumen}

Para superar rupturas del modelo educativo que influyen en la deserción en el sistema de educación universitario, acordamos realizar una intervención matriculando a los estudiantes indígenas y afrocolombianos admitidos en el 2006-II a los diferentes programas de ingeniería que ofrece la Universidad del Valle en los cursos de cálculo. La experiencia se da como un proceso de investigación-intervención que se diseñó e implementó con el objetivo de proporcionar una oportunidad, real y no solo legal a los estudiantes que ingresan por condición de excepción étnica a los planes de ingenierías, de acceder a los conocimientos científicos y tecnológicos de que se ocupa dicha universidad.

Palabras clave

Deserción; modelo educativo; contrato didáctico; evaluación.

\section{Integration policies for university education. The case of indigenous and Afro-Colombian people and the problem of dropout}

\section{Abstract}

To overcome the ruptures of the educational model that influence the desertion in the university education system, we agreed to carry out an intervention by enrolling the

\section{Contacto:}

Rafael Alberto Méndez Romero, rafael.mendez@urosario.edu.co, Edificio Cabal, Carrera 6 \# 12C-16, oficina 504 - Bogotá, Colombia 
indigenous and Afro-Colombian students admitted in 2006-II to the different engineering programs offered by the Universidad del Valle in the courses of calculation. The experience is given as an investigation-intervention process that was designed and implemented with the objective of providing an opportunity, real and not only legal to the students who enter the engineering plans due to ethnic exception condition, to access the knowledge scientific and technological aspects of the university.

\section{Key words}

Dropout; educational model; didactic contract; assessment.

\section{Introducción}

\section{La economía global y la educación}

El Sistema Educativo Colombiano adolece de un estado de crisis permanente y, en particular, la enseñanza de las matemáticas en lugar de contribuir al desarrollo de la inteligencia cumple una función de exclusión que actúa sobre la gran mayoría de la población la cual está destinada a formar parte de un amplio sector que vive del trabajo informal y en el mejor de los casos acceden a empleos técnicos pobremente remunerados.

Esta situación se generó, a nivel mundial, con el desarrollo de la producción masiva de bienes y servicios sustentada en los desarrollos tecnológicos que fueron posibles gracias a los conocimientos científicos desarrollados a partir del siglo XVII -en particular los Principia de Isaac Newton- y condujeron a la pauperización de los trabajos artesanales. De esta manera se produjo una ruptura del orden económico mundial que se expresó en la formación de países ricos poseedores de las patentes de la producción tecnológica y países pobres proveedores de materias primas. De acuerdo a esta organización la educación se ocuparía de educar una élite en buenas escuelas y centros de investigación reconocidos, y por otra parte educaría a la gran masa de la población en el aprendizaje de técnicas para realizar, de manera eficiente, tareas rutinarias en los diferentes campos del trabajo. Este modelo educativo se ha tornado insostenible. Se hace necesario realizar un giro del paradigma educativo hacia el desarrollo de la inteligencia en un marco inter y transdiciplinar, en condiciones de igualdad para todos los seres humanos, más que una educación centrada en la memorización de contenidos y desarrollo de destrezas como lo hace el paradigma dominante de la educación tradicional actual.

\section{Rupturas del modelo educativo universitario y la deserción}

El fracaso en los primeros cursos de matemática y su relación con los altos índices de deserción que se presenta en las instituciones de educación superior (IES) en Colombia, es problema viejo y en estudios del pasado (Grupo de Educación matemática de la Escuela Regional de Matemáticas, 1990; Álvarez \& Marmolejo, 1990) se consideró que las principales causas del bajo aprovechamiento de los primeros cursos universitarios respondían a cinco rupturas entre lo que ahora denominamos los tres polos del problema: Modelo Educativo, Formación Matemática de los alumnos y Condiciones de Estudio.

El Modelo Educativo (ME): Es la concreción de una teoría pedagógica. Comprende la estructura de contenidos, objetivos, medios, actividades y reglas que definen como se planifica, ejecuta y evalúa la enseñanza.

Determina una visión institucional del qué se enseña, cómo se enseña y cómo se concibe el aprendizaje de los alumnos. 
El modelo educativo tradicional dominante a nivel mundial está orientado por un paradigma didáctico que Yves Chevallard (2015) llama metafóricamente «Monumentalista» haciendo referencia a una enseñanza centrada en contenidos: «obras matemáticas» respuestas a cuestiones que se plantearon en el pasado. De esta manera, las problemáticas y el proceso de elaboración de las respuestas a las cuestiones se hacen invisibles en la enseñanza. Así, el docente es el protagonista de la clase, está «obligado» a explicar, validar y «transmitir» el saber institucionalizado. En consecuencia el alumno tiene pocas oportunidades de ser el constructor de la obra matemática, su papel será el de comprender, reproducir y aplicar las respuestas que le proporciona su profesor.

La Formación Matemática (FM): Es un sistema complejo adaptativo de comportamiento. Se construye, a lo largo de la vida del sujeto, por experiencias de todo tipo y se estructura en actitudes y valores; campos conceptuales, capacidades, habilidades y competencias intelectuales asociadas a la actividad matemática. Evoluciona por la maduración y el aprendizaje.

Determina tanto el ritmo de aprendizaje como las condiciones y restricciones que deben tomarse en consideración para la construcción de la, así llamada por Lev Vygotsky (1996, pp. 181-186), Zona de Desarrollo Próximo (ZDP) que favorece el aprendizaje de conocimiento matemático en un momento dado.

Aquí entendemos el aprendizaje como el producto de un proceso de autoorganización que resulta del comportamiento (i.e. el aprendizaje como la modificación de la experiencia por efecto del comportamiento) y APRENDER es adaptarse a situaciones nuevas.

Las condiciones de estudio (CE): Se refieren al capital académico y cultural: Hábitos y habilidades de estudio, tiempo dedicado a la actividad de estudio, ambiente sociocultural.

Determina el nivel de concentración y esfuerzo reales que alcanza el alumno en el estudio de un curso.

Rupturas entre el ME y la FM

- Ruptura I: El ME es retórico, rígido, centrado en contenidos y recargado. No reconoce la heterogeneidad de la FM de los estudiantes que ingresan a la Universidad

- Ruptura II: El ME No actúa sobre las deficiencias reales del estudiante y por lo tanto su efecto en la FM es restringido.

Rupturas entre la FM y las CE

- Ruptura III: Las CE limitan la evolución de la FM al ritmo que demanda el estudio en las IES.

Rupturas entre el ME y las CE

- Ruptura IV: La estrategia retórica del ME no provee formas de seguimiento de la actividad matemática del estudiante y en consecuencia tiene un bajo impacto en las CE.

- Ruptura V: El ME impone un tiempo didáctico que es diferente al ritmo de aprendizaje condicionado por las CE. El desfase entre el ritmo de exposición del Profesor y el ritmo del aprendizaje del alumno es consecuencia de las rupturas I y II. 


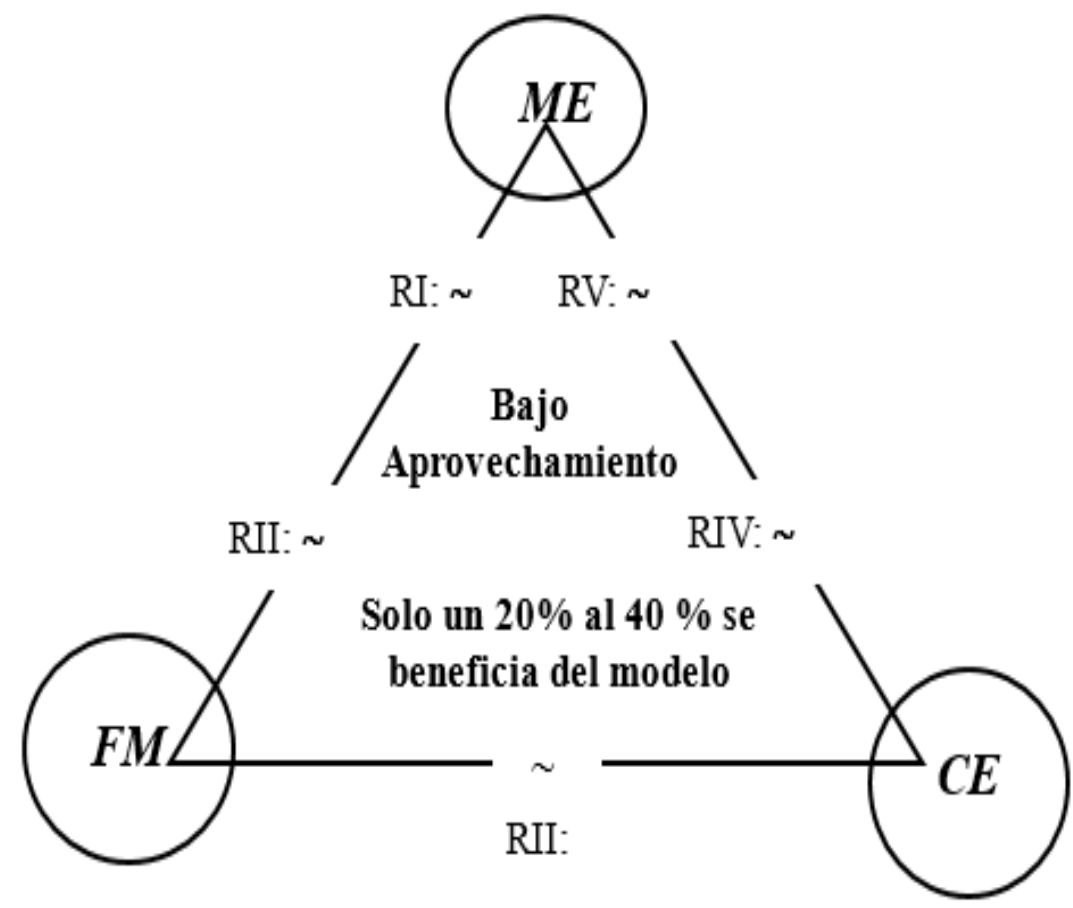

Figura No.1. Elementos determinantes del aprovechamiento estudiantil y las principales rupturas que originan estados de funcionamiento no deseables del sistema

Nuestra convicción y los estudios que hemos realizado en el pasado, como la experiencia de los Cursos Piloto, que es objeto de este artículo, nos indican que es posible enfrentar institucionalmente a estas rupturas y de esta manera no sólo disminuir la deserción sino potenciar el desarrollo de las competencias generales y profesionales de los egresados de la Educación Universitaria.

\section{Los Cursos Piloto como una respuesta a la tensión no deseable, desde una perspectiva basada en proyectos}

La apuesta por el aprendizaje basado en proyectos pone al estudiante en el centro y le hace enfrentarse a problemas de la vida cotidiana en donde, al estudiarlos y aproximarse a su solución, moviliza competencias y contenidos, interiorizando, así, saberes y generando nuevo conocimiento significativo.

En este tipo de aprendizaje, se hacen visibles tres principios constructivistas esenciales (Cocco, 2006):

1. El aprendizaje ocurre en un contexto específico, en cuanto "cada alumno estructura su conocimiento del mundo a través de un patrón único, conectando cada nuevo hecho, experiencia o entendimiento en una estructura que crece de manera subjetiva y que lleva al aprendiz a establecer relaciones racionales y significativas con el mundo" (Abbott y Terence, 1999).

2. Los estudiantes participan activamente en el proceso de aprendizaje, ya que se enfatiza en la construcción del aprendizaje, en lugar de la tradicional reproducción del mismo, máxime cuando la reflexión de las experiencias es un pilar fundamental 
en este enfoque. Acá, el matiz diferenciador es que los estudiantes planean, implementan y evalúan sus proyectos (Blank, 1997).

3. Los estudiantes alcanzan sus logros a través de interacciones sociales, su comprensión de lo aprendido (o por aprenderse) y el intercambio de conocimiento. De acá que las experiencias de aprendizaje de los estudiantes sean especialmente significativas (Wurdinger, 2007).

Aunque este tipo de aprendizaje está ligado con el aprendizaje basado en problemas (Helle, Tynjälä y Olkinuora 2006), sobre todo en el hecho de que ambos dan protagonismo al trabajo colaborativo, distan en que el aprendizaje basado en proyectos requiere un producto final de aprendizaje (Blumenfeld, Fishman, Krajcik, Marx y Soloway, 1991), a la manera de artefacto concreto, que representa la nueva comprensión del estudiante, el conocimiento adquirido y explicitado, y sus actitudes a la tensión de investigación que puede presentarse en distintos formatos (Holubova, 2008).

Dado que es un tipo de aprendizaje centrado en el estudiante, la autorregulación (Barak, 2012) es transversal a todas sus fases, haciendo que el estudiante sea autosuficiente especialmente en el establecimiento de metas (de aprendizaje), la planeación, organización y ejecución de estrategias que le permitan alcanzar objetivos vinculados a los proyectos.

Finalmente, para garantizar la eficacia de este tipo de aprendizaje, Mergendoller y Thomas (2005) proponen siete puntos a tenerse en cuenta: el primero tiene que ver con la correcta planeación de los tiempos; el segundo con todo el sistema de orientaciones anticipadas por parte del profesor, a propósito del diseño del proyecto; el tercero con la iniciación de los estudiantes en una cultura que decanta hacia la autogestión; el cuarto con la importancia del trabajo colaborativo vía grupos de trabajo; el quinto con la emergencia de asociaciones externas, es decir, la vinculación con otras personas fuera del aula (padres, profesores, amigos); el sexto con la incorporación de tecnologías para la comprensión, análisis y presentación de resultados, máxime en el momento histórico en el que nos encontramos; y finalmente, el séptimo, hace referencia a la incorporación de una gran variedad de métodos de evaluación que permitan observar, de cierta manera, el alcance cognitivo de los estudiantes a lo largo del proyecto.

Teniendo esto en cuenta, a continuación mostramos los detalles de lo que llamamos respuesta posible, como propuesta para solucionar la ruptura

Evidencia empírica de una respuesta posible: la experiencia de los cursos piloto de matemáticas.

Como ya se ha señalado, el problema del bajo aprovechamiento de los primeros cursos de matemáticas y su contribución a la alta tasa de deserción que se presenta en el Nivel Universitario -el promedio acumulado de las cohortes de 1998-I a 2003-I en el Nivel Universitario fue del $42,2 \%$ según informe del MEN (2009) - se relaciona, entre otros factores, con las condiciones y restricciones que impone el ME que funciona de manera ciega respecto a la FM y las CE de la población objeto.

Es así como las instituciones universitarias han desarrollado diferentes estrategias como la de nivelación, acompañamiento de monitores, oportunidades para la repetición de cursos, etc. a fin de disminuir la deserción, con muy pobres resultados. Esta situación es más dramática en el caso de los estudiantes que ingresan por régimen de excepción. Nuestra posición es que esta situación puede mejorar sin incrementar los costos de los programas, bajo ciertas condiciones institucionales que permitan superar las rupturas del ME y reorientar la acción educativa que se expresa en su funcionamiento. Para probar esta afirmación decidimos asumir el reto que nos planteó la directora del programa «Universidad 
y Culturas», de trabajar con los indígenas y afrocolombianos que ingresaron por régimen de excepción en el II semestre de 2006 con la meta de bajar la deserción de esta población que en los dos primeros semestres históricamente superaba el $60 \%$.

\section{Estrategia didáctica socioconstructiva}

\section{Respecto a las prácticas de enseñanza}

Nosotros propusimos abandonar el papel, que el paradigma de la didáctica monumentalista le asigna al profesor, de transmitir obras matemáticas y, a cambio, asumir la función como diseñador de situaciones «adidácticas» o «problémicas» que demandan la acción del estudiante y generan la necesidad de construir respuestas a cuestiones cuya solución sólo es posible por un proceso creativo de conocimientos necesarios para alcanzar el éxito de la acción, proceso que está a cargo del alumno y que se apoya, según la teoría de situaciones de Guy Brousseau (1997), con la «mediación didáctica» del profesor; cuyas acciones, se orientan a gestionar «situaciones didácticas» a fin de producir «devoluciones de problema» a los alumnos con el objetivo de provocar conflictos cognitivos entre el conocimiento del alumno y las resistencias que opone la situación que obligan a construir nuevos posibles, para satisfacer lo que ahora se ve como necesario, y luego ponerlos a prueba y validarlos primero en la acción y luego en la reflexión conceptual que los articula con el conocimiento formalizado en relación con el marco teórico-formal de las matemáticas y así construir nuevo -para el estudiante- conocimiento que es reconocido como un saber en la «institucionalización» que realiza el profesor.

En esta nueva estrategia, la evaluación es el "sistema nervioso" de la organización de las actuaciones adidácticas a cargo del estudiante y didácticas a cargo del profesor. Ahora es sistémica-formativa y permanente: se avalúan los resultados de la interactividad que se orienta a mediar los procesos cognitivos de los actores en el marco del funcionamiento de los subsistemas Alumno-situación adidáctica, Profesor-situación didáctica, que son constitutivos del sistema didáctico que los engloba.

\section{Dos Instrumentos de mediación: Guías de Apoyo Teórico y Guías de Trabajo}

El curso se desarrolló en torno a dos tipos de Guías: de "Apoyo Teórico" y guías de "Trabajo". Desde una perspectiva constructivista, la gestión de los diferentes temas del programa se piensa en torno a problemas - prácticos y teóricos- que hacen necesario un saber matemático específico (C), que no poseen los estudiantes, pero que es posible alcanzar cuando el alumno trabaja sobre un conjunto fundamental de situaciones matemáticas $S(C)=\left\{S_{1}, S_{2}, S_{3}, \ldots, S n\right\}$. El estudiante deberá aplicar sus conocimientos actuales $(\hat{C})$, que en primera instancia son insuficientes para resolver cada una o algunas de las situaciones Si para $i=1,2, \ldots, n$. Esta limitación de $\hat{C}$ plantea un problema $(P)$ al alumno como consecuencia de la diferencia entre el saber $C$ necesario y los conocimientos $\hat{C}$ disponibles en el momento de iniciar la secuencia didáctica $(P=C-\hat{C})$; el problema se resuelve cuando $\hat{C}$ iguala a $\mathrm{C}$.

La afirmación, a priori, que acabamos de hacer respecto a que a los estudiantes les es "posible alcanzar" el conocimiento $C$ es relativa al estado de los conocimientos $\hat{C}$ y a la mediación del profesor y de los orientadores del taller. Se trata, en la terminología de Lev Vygotsky (1996, pp. 181-186), de construir una «Zona de Desarrollo Próximo»-distancia cognitiva entre lo que el sujeto puede hacer a solas y lo que realiza con la ayuda de un experto- en la que: "[...] los conceptos espontáneos, faltos de control consciente y volitivo, encuentran dicho control, ..., con la cooperación entre el niño y los adultos" (Ídem, p. 185); en nuestro caso el alumno y los profesores, en torno a S(C). 
En el escenario que acabamos de describir, La Guía de Trabajo define la estructura de la secuencia didáctica para enseñar $C$ en torno al conjunto $S(C)$. El papel de la Guía de trabajo es el de ser un instrumento que potencialmente puede mediar, las acciones didácticas del profesor en el proceso de enseñanza y las acciones de los estudiantes en su aprendizaje, cuando en torno al saber que implícitamente aparece como necesario para el éxito de la tarea se articulan las acciones del profesor con las acciones de los estudiantes -interactividad (c.f. Coll, C. 1995) - buscando influir en los procesos cognitivos del otro para construir dominios de significados socialmente compartidos -aprendizaje. Por otro lado, dado el carácter potencial de la mediación, la Guía de Apoyo Teórico cumple dos funciones. La primera es proporcionar saberes matemáticos de acuerdo con las necesidades técnicas que demanda la construcción de $\mathrm{C}$ y, la segunda, proporcionar nuevas situaciones adidácticas que se ajusten más al estado de conocimientos del alumno -ligeramente por encima de los conocimientos actuales- cuando las situaciones propuestas en la Guía de Trabajo superan el desarrollo potencial del alumno. En resumen, el profesor ajusta sus acciones -elaborando Guías de Apoyo- de acuerdo con los análisis de los resultados que producen los alumnos al responder por la Guía de Trabajo, en contraste con los resultados esperados de acuerdo con ciertos supuestos a priori que definieron el tipo de situaciones.

La Guía de trabajo es el instrumento que potencialmente puede ser mediador de las acciones y, para que realmente lo sea, se complementa con las Guías de apoyo, para ajustar las acciones de acuerdo al estado real de conocimientos de los estudiantes.

Nuestra estrategia socioconstructivista (Delgado, 1998) se diferencia del modelo tradicional -que fundamenta la enseñanza en la lógica de la explicación en dos aspectos fundamentales:

1. La teoría y las técnicas matemáticas no son un producto acabado u obra muerta que se expone al estudiante para que las aprenda y en algún momento las aplique a la solución de situaciones fuera del aula.

2. Se obliga a un cambio de las actividades tradicionales del profesor y del estudiante: el primero no es más el poseedor del saber que centra su actividad en la administración de "buenas explicaciones", sino que, en el marco socioconstructivista, es más un diseñador y gestor de situaciones adidácticas relacionadas con el conocimiento objeto de la enseñanza, que media los procesos de aprendizaje; y el segundo, pasa de ser un receptor del conocimiento acabado, transformado y modelado por la explicación del profesor, a ser un sujeto que desarrolla una actividad de estudio en la que construye activamente su propio conocimiento con el objetivo de aprender matemáticas.

El primer aspecto se relaciona fundamentalmente con la actividad del profesor y en particular con el diseño de La Guía de trabajo y la Guía de Apoyo Teórico. Como ya se dijo más arriba, la Guía de trabajo expresa el conjunto fundamental de situaciones $\mathrm{S}(\mathrm{C})$ que se construye considerando las variables didácticas -valores de la situación que obligan a un cambio de estrategia en una situación adidáctica S- que se expresa en un estado de conocimiento mejor adaptado a la situación.

Estas variables didácticas se determinan a partir de:

- un estudio de la naturaleza del conocimiento matemático - dimensión epistemológica;

- el estado de conocimiento actual de los estudiantes - dimensión cognitiva; y 
- la gestión de los medios y procesos de enseñanza y aprendizaje - dimensión didáctica.

El segundo aspecto se refiere tanto a la actividad de gestión del profesor como a la actividad constructiva del estudiante. Decimos que "se obliga a un cambio de las actividades tradicionales..." porque cuando el profesor evita proporcionar, directamente, el conocimiento que es necesario para resolver la situación adidáctica, el alumno ineludiblemente tendrá que actuar usando su propio repertorios de conocimientos para alcanzar el éxito en la tarea -situación adidáctica de acción (SA)-; y luego, cuando se ve obligado a compartir con los otros y comunicar el producto de su acción, verbaliza y simboliza sus acciones -situación adidáctica de formulación (SF)- y, dado que son inevitables las demandas de explicaciones o cuestionamientos de sus pares, deberá tratar de convencer sobre la validez de sus resultados -situación adidáctica de validación (SV). En este conjunto de momentos o situaciones adidácticas de la enseñanza, el profesor toma cierta distancia, pero está atento para hacer que las situaciones adidácticas evolucionen de acuerdo con el aprendizaje del saber matemático C propuesto.

El funcionamiento adidáctico es posible si el profesor genera un marco didáctico que tiene como función la regulación de la situación adidáctica. El profesor en situación didáctica observa las acciones de los estudiantes y en concordancia con ellas actúa produciendo retroalimentaciones (positivas o negativas) para llenar lagunas -carencia de ciertos conocimientos necesarios para alcanzar $C$ (pero nunca el conocimiento $C$ que es el objeto de aprendizaje) - o para generar conflictos cognitivos con respecto a los conocimientos obstáculo que estén presentes en el estudiante -situación didáctica de devolución de problema (SD). También el profesor actúa para reconocer que el conocimiento construido por el estudiante es un saber matemático de pleno derecho -situación de institucionalización (SI). Estas acciones del profesor siempre están articuladas con las acciones del aprendiz sobre la situación adidáctica y es una respuesta a ellas para provocar el cubrimiento de una laguna o la superación de un conocimiento obstáculo.

En resumen se espera que como resultado de la operacionalización de los dos aspectos del modelo, el conocimiento $C$ sea el resultado de satisfacer las variables de la siguiente función de conocimiento:

$$
\mathrm{C}=\mathrm{SA}+\mathrm{SF}+\mathrm{SV}+\mathrm{SD}+\mathrm{SI}
$$

$\mathrm{Y}$, en consecuencia, cada Guía define la estructura de la secuencia didáctica y cumple la función de instrumento mediador, de las acciones didácticas del profesor en el proceso de enseñanza y de las acciones de los estudiantes en su proceso aprendizaje.

\section{Cómo se usaban las guías}

En la primera semana del curso de cálculo I tomamos conciencia de la inmensa brecha entre el estado de la formación matemática de los estudiantes y las demandas que planteaba el programa del curso, que pretendíamos cubrir en siete Guías. Esto obligó a elaborar nuevas Guías que se ajustaran a las condiciones de formación matemática de los estudiantes; por lo cual, más que trabajar sobre contenidos buscábamos incidir en el desarrollo de ciertas competencias para:

- utilizar lenguaje matemático,

- razonar matemáticamente e

- imaginar mundos posibles 
De esta manera, nuestro objetivo de fondo en la gestión de cada una de las guías consistió en transformar por el desarrollo de la actividad conjunta en torno al objeto de aprendizaje la tendencia a pensar la actividad de estudio de las matemáticas como aprendizaje de fórmulas y algoritmos.

En el curso de cálculo II continuamos utilizando la misma metodología que en el primer curso, la cual obligaba a los estudiantes a trabajar antes de la clase las situaciones de la Guía y a usar el encuentro con los asistentes de docencia en el Taller $(2 \mathrm{~h} / \mathrm{s})$ para aclarar dudas y recibir retroalimentaciones a fin de realizar su obra matemática. En las clases (dos, espaciadas por un día, de $2 \mathrm{~h} / \mathrm{s}$ cada una), el profesor también trabajaba con los estudiantes a partir de sus preguntas sobre el tema asignado en la Guía, y trataba de desarrollar una interactividad para afectar los procesos cognitivos que orientan las acciones de estos. Buscaba que se produjeran los aprendizajes, pero sin dar respuestas directas que fueran solución de la situación.

Con estas ayudas el estudiante desarrollaba una producción escrita sobre situaciones de la Guía previamente asignadas la tarea, y la entregaba semanalmente a los asistentes de docencia para su corrección. Sin embargo, es conviene subrayar que mediando el aprendizaje bajo la lógica de la construcción y no con la lógica de la explicación necesariamente se avanza más lentamente: la comprensión es un proceso lento en el que se siembra la semilla del entendimiento y para que se convierta en fruto hay que realizar un cuidadoso seguimiento e invertir tiempo.

El profesor diseñaba una prueba corta semanal sobre los puntos de la tarea, y se aplicaba el mismo día en que los estudiantes entregaban la tarea. En el encuentro siguiente a la entrega de las tareas, los asistentes de docencia devolvían a los estudiantes las tareas corregidas y comentadas, así como los resultados de la prueba corta. En este momento, los asistentes de docencia, tomando en consideración los resultados logrados, y las retroalimentaciones escritas por ellos en cada tarea o prueba, discutían con los alumnos los puntos en los que se habían detectado aprendizajes deficientes. Luego, los asistentes de docencia informaban al profesor, en un formato especial, cuáles habían sido los resultados en términos de lagunas y obstáculos más frecuentes presentes en los estudiantes. Esta información era la base con la cual el profesor planificaba el trabajo de la semana siguiente.

Es evidente que la metodología sobre la cual se fundó todo el curso -la manera de concebir las clases centrada en la interactividad entre el profesor y los estudiantes en torno a los objetos de aprendizaje; los talleres basados en lo que según las tareas y pruebas cortas veíamos que aún no se había comprendido; la retroalimentación escrita como comentarios a sus procesos de razonamiento matemático, empleo del lenguaje matemático en los procesos de construcción, formulación y validación de los conocimientos matemáticos que se observaba en las tareas, y la exigencia de toma de conciencia del error que este modo de proceder le plantea a los alumnos como fuente y condición necesaria para el aprendizajerompía el contrato didáctico sobre el cual se había basado toda su escolaridad.

El equipo de asistentes y el profesor eran conscientes de las rupturas del contrato didáctico necesarias para avanzar en el objetivo central del curso piloto de disminuir la deserción de los planes de ingeniería y al mismo tiempo plantear niveles altos de comprensión de las matemáticas. De esta manera, parte del trabajo del equipo docente era resolver con el diálogo razonado las crisis y superar las rupturas actualizando las obligaciones implícitas del contrato.

\section{El contrato didáctico}


El contrato se fundamenta en seis principios, propios de un proceso de enseñanzaaprendizaje fundamentado en una epistemología constructivista radical. Ellos son:

- Primero: Sólo interesa aquello que es fundamental y básico.

- Segundo: La necesidad es generadora de conocimiento.

- Tercero: La reflexión sobre el error es importante.

- Cuarto: Interesa la superación del error.

- Quinto: Se aprende haciendo.

- Sexto: No se aprende a solas.

El objeto del contrato, cuyas clausulas en su mayor parte son implícitas, es la enseñanza y el aprendizaje del saber matemático y este contrato, que obliga al profesor a enseñar y al alumno a aprender, regula el funcionamiento del curso -sistema didáctico. Las rupturas del contrato generan crisis que se toman como verdaderas oportunidades de progresar y superar estados de funcionamiento del sistema didáctico que impiden o limitan el acceso al nuevo conocimiento.

En el curso de cálculo II, alrededor de la sexta semana se viven estas rupturas, donde los estudiantes exigen "clases magistrales...", "ir un poquito más rápido..." y reclaman al profesor por su flexibilidad con los que "no saben" y exigen que "se les enseñe las matemáticas sin física...". Estas rupturas, que se encuentran registradas, se explican a continuación:

Respecto a las clases magistrales y los ritmos de exposición versus el ritmo de aprendizaje

Explicar la teoría y avanzar más rápidamente: “... Profe y usted no puede hacer clases un poquito más magistrales..." donde "... se explique cómo hacer la cosas, para que nosotros podamos aprender" y se pueda “... ir un poquito más rapidito... pero no devolverse...".

El profesor explica que se avanza de acuerdo al ritmo de aprendizaje de los estudiantes y proporciona algunos ejemplos de conceptos que en la misma clase no han sido comprendidos por los alumnos a pesar que se han trabajado (medida de ángulos) o y discutido en las clases anteriores.

Aunque el profesor desde el primer día de clase explicó que la empresa del aprendizaje de conceptos científicos es colectiva, no todos los estudiantes asumen real y eficientemente su actividad de estudio. Es por esta razón que el profesor se centra en aquellos alumnos que aún no asumen la responsabilidad de la tarea y produce devoluciones de problemas negándose a proporcionar las respuestas que debería elaborar el alumno, y esto es causa de malestar en los estudiantes. No se comprende por qué el profesor no explica la solución del problema para avanzar más rápidamente — parte de la estrategia socioconstructivista es negarse a ello y las intervenciones magistrales se reservan para el momento de institucionalización de los conocimientos producidos por los alumnos. En esa situación didáctica el profesor explica, relaciona, y reconoce el valor del conocimiento obtenido por el alumno y señala su posición como un Saber matemático al tiempo que muestra las implicaciones y posibilidades de aplicación de este conocimiento. Este proceder nos lleva a la siguiente observación de los alumnos.

\section{Respecto a la regulación de la autoridad del profesor}

Demanda por la regulación de la autoridad del profesor: “....A mí me parece que usted es muy flexible con nosotros. $Y$ es culpa de nosotros, pero también es culpa suya. Uno sabe que no importa si no estudia, porque si el profesor va a volver a hablar de lo mismo la próxima clase ¿uno para qué estudia?” 
Este reclamo es justo y bien planteado. El fenómeno estaba entre las expectativas del equipo docente. Transformar las prácticas individuales de estudio en prácticas de responsabilidad compartida requiere de un proceso de toma de conciencia a partir de la propia experiencia y de la presión de la sanción social. La forma como se concreta es tratando el problema de la actividad de estudio no por los resultados buenos de algunos estudiantes que trabajan y comprenden a ritmos adecuados, sino a partir de los casos en que sucede lo contrario y dar toda la atención de la clase a ellos sin menoscabo de que quienes han hecho su trabajo no mejoren y progresen en la comprensión de los que han trabajado, así 'para ellos aparentemente "están perdiendo el tiempo".

Esta manera de proceder es un mecanismo de influencia educativa. El estudiante que no realiza el trabajo diario no puede escapar al interrogatorio y es sometido a situaciones que buscan ayudarlo en su tarea.

\section{Respecto a los contextos}

No trabajar problemas en contexto: "Queremos que nos explique las matemáticas sin física..." "Entender las funciones con la física se nos hace más complejo, hay que saber física y funciones...," "Se le hace a uno más difícil comprender funciones en matemáticas ahora como no se hará más difícil a uno comprenderlas en física"

Esta solicitud "Queremos que nos explique las matemáticas sin física..." parece contradecir la petición contraria que también se escucha en el aula de matemáticas:

“¿Por qué no nos explica eso de los espacios n-dimensionales con ejemplos en el espacio de tres dimensiones y palabras que podamos entender? “

Es bien interesante esta paradoja. Ella refleja dos percepciones distintas respecto a las relaciones entre las matemáticas y el mundo real.

La primera, respecto a las aplicaciones de las matemáticas. La forma como tradicionalmente los alumnos se relacionan con las matemáticas en el aula -como fórmulas ya elaboradas- y sus aplicaciones -sustituciones de los datos, proporcionados en el enunciado de un "problema", en la fórmula para encontrar una incógnita-conduce a los estudiantes a creer que las matemáticas se refieren a un mundo diferente y desconectado con el de sus aplicaciones prácticas.

El reto que se planteó el equipo docente fue entonces articular la experiencia de los alumnos en el dominio de la acción práctica sobre los problemas reales en situaciones del mundo físico, económico, social, etc., con el pensamiento que actúa sobre aquellos mismos objetos y situaciones pero ahora operando sobre proposiciones formales, modelos matemáticos; apoyándose en diversos lenguajes para ayudar a los procesos de la mente en el momento de aplicar, formular y validar los conocimientos que permiten sortear con éxito los problemas.

La segunda percepción, respecto a la relación de lo real con los modelos abstractos y el lenguaje en que ellos se expresan. Por una parte, por más que se esfuerce el profesor en traducir sus modelos matemáticos a palabras del lenguaje ordinario sólo logrará comprensiones parciales y ello porque las matemáticas no son sólo un lenguaje sino que son lenguaje, más razonamiento y más invención. Perder uno de estos elementos del foco de la enseñanza y del aprendizaje es desnaturalizar las matemáticas y, lo que es más grave reducir su maravilloso poder para ayudar a comprender el mundo. El problema que se plantea es cómo lograr recrear en el aula una matemática viva que permita desarrollar su verdadera naturaleza. Nuestra apuesta es que si se dispone de cierta conciencia teórica de la experiencia del mundo físico -y este es el objetivo de los cursos de física- ella ayuda a 
intuir y apreciar ciertos conceptos y teoremas matemáticos que de otra manera sería muy difícil imaginar y valorar.

Por supuesto que los razonamientos matemáticos que se han desarrollado son de gran utilidad para los físicos. Por otra parte, también a veces los razonamientos de los físicos son de gran utilidad para los matemáticos. (Feynman, 1980, p. 43)

$\mathrm{Y}$, desde el punto de vista educativo, es importante explotar esta necesidad mutua de los saberes, desarrollar el pensamiento causal que se deriva de los objetos físicos y el pensamiento lógico-matemático que lo interpreta y organiza.

El drama que expresan los estudiantes en la afirmación:

"Se le hace a uno más difícil comprender funciones en matemáticas ahora como no se hará más difícil a uno comprenderlas en física"

Refleja la presión de que sobre ellos ejerce la estrategia educativa que nos planteamos en el curso y se discutió con ellos sobre las razones de este manera de actuar, advirtiendo que es una empresa difícil y necesaria, pero no insuperable.

\section{Respecto a las expectativas del equipo docente}

En el curso de Cálculo I sólo se construyó el concepto de estructura algebraica de cuerpo ordenado y completo de los números reales y esperábamos que el desarrollo de la competencia matemática lograda en el semestre permitirían, en el segundo semestre, cubrir los temas que quedaron pendientes de cálculo I y los de cálculo II. Sin embargo existían reservas respecto al cumplimiento de nuestro compromiso de responder por el cubrimiento de los contenidos de los dos cursos; por tres razones: 1) las expectativas respecto al nivel de formación matemática de esta población pecó por optimista, no obstante que preveíamos que el nivel era bajo, 2) el atraso en los temas de cálculo I fue muy significativo y 3) por la lentitud en que se modificaban los métodos de estudio de los alumnos y la resistencia de ellos al cambio.

Por tanto, el equipo siempre fue consciente del atraso en los contenidos y los problemas curriculares que ello ocasiona. Sin embargo, se estaba seguro que la experiencia estaba transformando de forma lenta pero segura la manera como los alumnos se relacionan con las matemáticas y con otros saberes, lo que podría subsanar en parte los desfases curriculares en el corto plazo, si se contaba con ayudas puntuales del profesor y los asistentes de docencia, para cubrir la parte algorítmica de la matemática necesaria en cursos como el de física.

\section{Respecto a las prácticas de estudio}

Con relación a la transformación de las prácticas de estudio, el alumno pasa de ser un receptor de soluciones acabadas para los problemas que en algún momento de la historia se plantearon los matemáticos y formalizaron en axiomas, definiciones, teoremas y algoritmos que él debe memorizar y cuyo funcionamiento él imita del modelo que proporciona las presentaciones y explicaciones del profesor; a ser, un constructor de su propio conocimiento matemático resolviendo problemas creativos cuyas restricciones, en relación con los conocimientos que libremente pone en juego el alumno, hacen que cierto conocimiento sea necesario para alcanzar el éxito.

Esta empresa, de ser constructor de su propio conocimiento, le demanda invertir tiempo en lo que se llama período de familiarización con los elementos relevantes de la situación que lleva a reconocer la existencia de un problema y plantear las cuestiones cuyas respuestas la permiten resolverlo. Luego es necesario realizar un duro trabajo en el que el alumno utiliza su repertorio de conocimientos y fracasa. Según los expertos y los testimonios de los 
mismos matemáticos, se sigue un período de incubación en el cual trabaja el inconsciente y termina cuando, como dice Poincaré (1913), este trabajo se manifiesta en un "momento repentino" de "iluminación" en el cual la solución aparece «como si surgiera de la nada» y finalmente un último periodo de verificación en el cual los resultados, que la iluminación presenta sólo groso modo, se enuncian con precisión: “[,,,] los cálculos efectivos, que requieren disciplina, atención, voluntad y por tanto, conciencia, dependen del segundo período de trabajo consciente que sigue a la inspiración.... [... inseparable de la primera, la verificación.» (Hadamard, 1947., pp. 103-104)

La estrategia que orienta las acciones del alumno y del profesor entorno de la construcción de conocimiento hace necesaria cierta flexibilidad en el manejos de los tiempos oficiales asignados para cubrir las temáticas de los programas de tal manera que sea posible acompasar los contenidos a los tiempos y ritmos de aprendizaje de los estudiantes, a medida que se operan ciertas transformaciones en su formación matemática y sus concepciones sobre el aprendizaje y sobre las matemáticas -concepciones que, en la mayoría de los estudiantes, son negativas y muy arraigadas por la cultura que se desarrolla en las aulas de matemáticas tradicionales.

\section{Conclusiones}

La propuesta de los cursos piloto logró disminuir notablemente los índices de deserción y produjo un cambio en la comprensión de la manera en cómo debería enseñarse (y aprenderse) las matemáticas, particularmente con este tipo de poblaciones vulnerables. Nuestro reto consistió en integrar al aula de matemáticas aspectos como el asombro y la invención, la intuición y la validación, el razonamiento y la lógica, la predicación y los conceptos, los juicios y los lenguajes matemáticos, bajo el supuesto que estos aspectos son constitutivos de la actividad de estudio que realiza tanto el matemático como los estudiantes que aprenden matemáticas. Tales aspectos son necesarios para la creación de nueva matemática.

\section{Referencias}

Abbott, J., \& Terence, R. (1999). Constructing Knowledge and Shaping Brains. Recuperado de The 21st Century Learning Initiative: http://www.21learn.org/

Álvarez, J \& Marmolejo, M (1990). Sobre el Bajo Aprovechamiento Estudiantil en los Primeros Cursos de Matemáticas en la Universidad del Valle. Matemáticas: Enseñanza Universitaria. Vol. 1, Nº 2, pp. 85-100.

Barak, M., \& Asad, K. (2012). Teaching image-processing concepts in junior high schools: Boys' and girls' achievement and attitudes towards technology. Research in Science \& Technological Education, 30, 81-105.

Blank, W. (1997). Authentic instruction. In W.E. Blank \& S. Harwell (Eds.), Promising practices for connecting high school to the real world (pp. 15-21). Tampa, FL: University of South Florida.

Blumenfeld, P., Fishman, B. J., Krajcik, J., Marx, R. W., \& Soloway, E. (2000). Creating usable innovations in systemic reform: Scaling up technology-embedded project-based science in urban schools. Educational Psychologist, 35, 149-164.

Brousseau G. (1997), Theory of Didactical Situations in Mathematics. Didactique des mathematiques, 1970-1990. Edited and translated by: Nicolas Balacheff, 
Martin Cooper, Rosamund Sutherland, y Virginia Warfield (eds. y trad.). Kluwer Academic Publishers. London.

Chevallard, Y. (2015). Teaching Mathematics in Tomorrow's Society: A Case for an Oncoming Counter Paradigm. En: The Proceedings of the 12th International Congress on Mathematical Education. Springer International Publishing. .Seoul, Korea, pp 173187

Chevallard, Y., Bosch, M., \& Gascon, J. (1997). Estudiar matemáticas. El eslabón perdido entre la enseñanza y el aprendizaje. Barcelona: I.C.E.-Horsori.

Cocco, S. (2006). Student leadership development: The contribution of project-based learning (Tesis de máster no publicada). Royal Roads University, Victoria, BC, Canada.

Coll, C., Colomina, R., Onrubia, J. y Rochera, J. (1995). Actividad Conjunta y Habla. En Fernández B. \& Melero Z. M. (compiladores), La interacción social en contextos educativos. Siglo XXI. Madrid, pp. 193-326.

Delgado, C. (1998). Estudio Microgenético de Esquemas Conceptuales Asociados a Definiciones de Límite y Continuidad en Universitarios de Primer Curso. Tesis Doctoral. Publicaciones Universidad Autónoma de Barcelona. España.

Grupo de Educación matemática de la Escuela Regional de Matemáticas (ERM), (1990). El problema del bajo aprovechamiento estudiantil en los primeros cursos universitarios de matemáticas. Matemáticas: Enseñanza Universitaria. Vol 1, No 1, pp. 51-58

Hadamard, J., (1945), The Psychology of Invention in the Mathematical Field., Princeton University Press, (page references are to the Dover edition, New York 1954). Versión castellana: La psicología de la invención en el campo matemático. Espasa-Calpe, Buenos Aires, 1947.

Helle, L., Tynjälä, P., \& Olkinuora, E. (2006). Project-based learning in post-secondary education - theory, practice and rubber sling shots. Higher Education, 51, 287-314.

Holubova, R. (2008). Effective teaching methods - project-based learning in physics. USChina Education Review, 12, 27-35.

Mergendoller, J. R., \& Thomas, J. W. (2005). Managing project based learning: Principles from the field. California: Buck Institute for Education.

MEN. (2009). Deserción estudiantil en la educación superior colombiana. Metodología de seguimiento, diagnóstico y elementos para su prevención. Ministerio de Educación Nacional. Bogotá.

Piaget, J. (1976) Le Comportament, Moteur de l'Évolution. Gallimard. Paris. Versión castellana: El comportamiento, motor de la evolución. Ediciones Nueva Visión, 1977. Buenos Aires.

Poincaré, H., (1908). L'invention mathématique. Conferencia presentada en el Institut général de psychologique, el 23 de mayo de 1908. Reproducida en la Revue générale des sciences pures et appliquées. 19 (1908), pp. 521-526. Versión consultada: Science et méthode, Flammarion, Paris, 1908; 1918, pp. 24-34.

Vygotski, L. S. (1930). El desarrollo de los procesos psicológicos superiores. Crítica. Barcelona, 1996. 
Wurdinger, S., Haar, J., Hugg, R., \& Bezon, J. (2007). A qualitative study using project-based learning in a mainstream middle school. Improving Schools, 10, 150-161. 